\title{
Closed (Blunt) Compared to Open (Penetrating) Pulmonary Contusion-A National Trauma Data Bank Review
}

\author{
${ }_{1}^{1}$ Gerd D Pust, ${ }^{2}$ Louis Pizano, ${ }^{3}$ Tanya Zakrison, ${ }^{4}$ Valerie Hart, ${ }^{5}$ Joyce Kaufman, ${ }^{6}$ Antonio Marttos, ${ }^{7}$ Rishi Rattan, \\ ${ }^{8}$ Howard Lieberman, ${ }^{9}$ Gabriel Ruiz, ${ }^{10}$ Edward Lineen, ${ }^{11}$ George D Garcia, ${ }^{12}$ Mauricio Lynn, ${ }^{13}$ Carl Schulman, \\ ${ }^{14}$ Patricia M Byers, ${ }^{15}$ Danny Sleeman, ${ }^{16}$ Enrique Ginzburg, ${ }^{17}$ Nicholas Namias
}

\begin{abstract}
Introduction: Pulmonary contusion is a common injury and may occur in blunt or penetrating trauma patients. These injury mechanisms may have a distinct pathophysiology, differ in the out come and may require individualized treatment. The purpose of this study is to compare these two mechanisms of pulmonary contusion in respect to outcome and clinical management.
\end{abstract}

Methods of Research: The 2012 National Trauma Data Bank (NTDB) was queried for open and closed pulmonary contusion utilizing ICD-9 codes 861.31 and 861.21. Data for both groups were analyzed with t-test for numerical data and chi-square for categorical data utilizing SPSS ${ }^{\mathrm{TM}}$ and VassarStats ${ }^{\mathrm{TM}}$.

Results: A total of 12,884 patients with pulmonary contusions were identified. The closed pulmonary contusion was present in 12,329 patients, open pulmonary contusion in 555 patients. Patients with closed pulmonary contusions were older with a mean age of $38.00 \pm 22.23$ versus $30.58 \pm 12.88$. Patients with closed pulmonary contusion had a higher injury severity score (ISS) $21.60 \pm 0.22$ versus $18.64 \pm 1.08$, $p=0.001$. Closed pulmonary contusion was associated with increased ventilator days $3.09 \pm 0.13, p=0.052$, intensive care unit (ICU) days $5.11 \pm 0.15$ versus $4.01 \pm 0.69, p=0.003$ and hospital length of stay $0.65 \pm 0.25$ versus $9.37 \pm 0.99$, $\mathrm{p}=0.032$.

Conclusion: Blunt (closed) pulmonary contusion is more prevalent than open (penetrating) pulmonary contusion

\footnotetext{
${ }^{1}$ Assistant Professor of Surgery, ${ }^{2-12,13-16}$ Professor of Surgery,

${ }^{17}$ Professor of Surgery/Chief, Division of Trauma and Acute Care Surgery

1,2,4,5,7,8,10-15 Division of Trauma and Surgical Critical Care, The DeWitt Daughtry Family Department of Surgery Ryder Trauma Center/Jackson Memorial Hospital Miller School of Medicine University of Miami, Miami, Florida, USA

${ }^{3}$ Surgery, University of Miami Miami, Florida, USA

6,9,16,17Dewitt Daughtry Department of Surgery, Division of Trauma and Surgical Critical Care, Ryder Trauma Center/Jackson Memorial Hospital, Miller School of Medicine, University of Miami, Miami, Florida, USA

Corresponding Author: Gerd D Pust, Assistant Professor of Surgery, Division of Trauma and Surgical Critical Care, The DeWitt Daughtry Family Department of Surgery, Ryder Trauma Center/Jackson Memorial Hospital Miller School of Medicine University of Miami, Miami, Florida, USA, Phone: +1-305-5851180, E-mail: gpust@med.miami.edu
}

and is associated with a higher injury severity score (ISS). Closed pulmonary contusion shows a tendency towards more extended mechanical ventilation time and is associated with a longer ICU stay, hospital length of stay and mortality. These findings might be secondary to associated chest wall trauma and other organ system injuries or secondary to the volume size of involved lung parenchyma. A pulmonary contusion may be a different entity compared to penetrating pulmonary contusion with different patient demographics, care requirements and outcome. Additional research is required to better understand contributing factors, differences in pathophysiology and clinical management of these two different disease processes.

Keywords: Chest trauma, ICU, Lung, Mortality, Penetrating, Pulmonary contusion (blunt, closed, open)

How to cite this article: Pust GD, Pizano L, Zakrison T, Hart V, Kaufman J, Marttos A, Rattan R, Lieberman H, Ruiz G, Lineen E, Garcia GD, Lynn M, Schulman C, Byers PM, Sleeman D, Ginzburg E, Namias N. Closed (Blunt) Compared to Open (Penetrating) Pulmonary Contusion-A National Trauma Data Bank Review. Panam J Trauma Crit Care Emerg Surg 2018;7(2):102-107.

Source of support: Nil

Conflict of interest: None

\section{INTRODUCTION}

Pulmonary contusion is frequently seen in patients with thoracic trauma. ${ }^{1}$ The vast majority of pulmonary contusions are a result of blunt, closed chest trauma. However, pulmonary contusions are also seen with open chest trauma in patients with pulmonary gunshot wounds. Pathophysiology, management, and outcome of pulmonary contusion in blunt trauma patients are well understood and documented. ${ }^{1,2}$ In patients with penetrating trauma, the associated pulmonary contusion is a result of the missile shockwave and blast effect. In blunt chest trauma, pulmonary contusion is often associated with significant chest wall injuries including multiple rib fractures or flail chest segments. ${ }^{3}$ The volume of lung parenchyma involved in blunt trauma patients is often extensive, involving multiple lung lobes and not uncommonly both lungs. ${ }^{2,4}$ In contrast, pulmonary contusions associated with gun shot wounds are localized around the missile track, often limited to one or two lung lobes and involve frequently only one lung. Furthermore, the associated 
chest wall injury is often more localized, involving one or two ribs or may result in no rib fractures at all.

Differences in pathophysiology and injury mechanism between blunt and penetrating pulmonary contusion may result in different outcomes and may require a more specific clinical management based on injury mechanism. In fact, pulmonary contusion in penetrating chest trauma might be an entirely separate entity from the blunt pulmonary contusion.

The purpose of this study is to evaluate whether penetrating pulmonary contusion differs from blunt pulmonary contusion in an outcome, clinical management requirements, and length of intensive care unit and hospital stay. To answer this question, we reviewed the NTDB for closed and open pulmonary contusion patients and compared both groups with respect to demographic, clinical management and outcome data.

\section{METHODS OF RESEARCH}

A retrospective analysis of the 2012 NTDB was performed. The NTDB represents is an extensive database, which receives standardized datasets from a majority of trauma centers in the United States annually. The 2012 NTDB dataset contains 179,197 trauma patient incidents, which allows studying injury diagnosed with sufficient sample size. However, the NTDB also has some limitations in respect to data accuracy. Injuries are recorded based on ICD-9 codes, injury mechanism E-codes and procedure $\mathrm{P}$-codes and therefore data quality is mostly dependent on correct injury coding, and data entry by each participating institution. ICD-9 codes 861.21 for closed pulmonary contusion and 861.31 for open pulmonary contusion were utilized to identify the study population. File Maker Pro ${ }^{\mathrm{TM}}$ database software was used to query the NTDB files. Demographic, treatment and outcome data were analyzed utilizing SPSS ${ }^{\mathrm{TM}}$ and VassarStats ${ }^{\mathrm{TM}}$. Statistical analysis with t-test for numerical data and chi-square for categorical data was performed.

\section{RESULTS}

We identified a total of 12.884 patients with a pulmonary contusion in the 2012 NTDB Closed chest pulmonary contusion was present in 12.329 patients $(95,7 \%)$, whereas open chest pulmonary contusion associated with penetrating trauma was present less frequently, in 555 patients $(4.3 \%)$. Patients with closed pulmonary contusions were older with a mean age of $38.00 \pm 22.23$ versus $30.58 \pm 12.88$. In the closed pulmonary contusion group, $72.7 \%$ were male patients, and $27.3 \%$ were female patients. In the open pulmonary contusion group, $89.4 \%$ were male patients, and $10.6 \%$ were female patients. Patients with closed pulmonary contusion had a higher ISS $21.60 \pm 0.22$ versus $18.64 \pm 1.08, \mathrm{p}<0.001$.
Table 1: Demographic data of study groups.

\begin{tabular}{lll}
\hline & $\begin{array}{l}\text { Closed pulmonary } \\
\text { contusion group }\end{array}$ & $\begin{array}{l}\text { Open pulmonary } \\
\text { contusion group }\end{array}$ \\
\hline Number of patients & 12,329 & 555 \\
Age & $38.00 \pm 22.23$ & $30.58 \pm 12.88$ \\
Male/Female ratio & $72.7 \% / 27.3 \%$ & $89.4 \% / 10.6 \%$ \\
ISS & $21.60 \pm 0.22$ & $18.64 \pm 1.08$ \\
\hline
\end{tabular}

There was no difference in respiratory failure rate with need for mechanical ventilation in the closed pulmonary contusion group with $36.0 \%$ and open pulmonary contusion group with $33.9 \%, \mathrm{X} 2(1, \mathrm{~N}=12884)=0.91, \mathrm{p}=0.3401$. However, closed pulmonary contusion was associated with an increase in ventilator days $3.09 \pm 0.13$ versus $2.46 \pm 0.69, \mathrm{p}=0.052$, and ICU length of stay $5.11 \pm 0.15$ versus $4.01 \pm 0.69, p=0.003$. Hospital length of stay was longer for closed pulmonary contusion with $10.65 \pm 0.25$ versus $9.37 \pm 0.99, p=0.032$. Mortality was with $7.66 \%$ in the closed pulmonary contusion group higher compared $4.68 \%$ in the open pulmonary contusion group, $\mathrm{X} 2(1, \mathrm{~N}=12884)=6.35, \mathrm{p}=0.017$. The incidence of pneumonia complication was significantly higher in the closed pulmonary contusion group with $10.5 \%$ versus $6.8 \%$ in the open pulmonary contusion group, $\mathrm{X} 2(1, \mathrm{~N}+12884)=7.32$, $\mathrm{p}=0.0068$.

Table 2: Results closed pulmonary contusion group and open pulmonary contusion group

\begin{tabular}{llll}
\hline & $\begin{array}{l}\text { Closed pulmonary } \\
\text { contusion group }\end{array}$ & $\begin{array}{l}\text { Open pulmonary } \\
\text { contusion group }\end{array}$ & p-value \\
\hline $\begin{array}{l}\text { Need for } \\
\text { mechanical } \\
\text { ventilation }\end{array}$ & $36.00 \%$ & $33.90 \%$ & $\mathrm{p}=0.3401$ \\
$\begin{array}{l}\text { Ventilator } \\
\text { days }\end{array}$ & $3.09 \pm 0.13$ & $2.46 \pm 0.69$ & $\mathrm{p}=0.026$ \\
$\begin{array}{l}\text { ICU length of } \\
\text { stay }\end{array}$ & $5.11 \pm 0.15$ & $4.01 \pm 0.69$ & $\mathrm{p}=0.003$ \\
$\begin{array}{l}\text { Hospital } \\
\text { length of stay } \\
\text { Incidence of } \\
\text { pneumonia } \\
\text { Mortality }\end{array}$ & $10.65 \pm 0.25$ & $9.37 \pm 0.99$ & $\mathrm{p}=0.032$ \\
\hline
\end{tabular}

\section{DISCUSSION}

Pulmonary contusion is a common injury in thoracic trauma. We identified 12,884 patients with pulmonary contusion in the 2012 NTDB. The 2012 NTDB dataset contains 179,197 trauma incidents, and therefore, the observed incidence of pulmonary contusion in this databank is $7.2 \% .^{5}$ Blunt thoracic trauma resulting in closed pulmonary contusion is more common then open pulmonary contusion associated with pulmonary gunshot wounds. However, it is difficult to evaluate if open pulmonary contusion associated with pulmonary 
gunshot wounds is underreported in the NTDB. Reported diagnosis with gun shot wounds to the chest frequently include hemothorax, pneumothorax, and lung laceration, but failure to recognize associated pulmonary contusion, especially in the absence of a computer tomography scan may occur.

The results in our study demonstrate that closed pulmonary contusion compared to open pulmonary contusion is associated with an increase in ventilator days, length of ICU stay, length of hospital stay, pneumonia rate and mortality. The incidence of respiratory failure requiring mechanical ventilation was similar in both groups.

The difference of the clinical course, complication rate, and overall outcome might be multifactorial. Closed pulmonary contusion (Fig. 1) requires a significant blunt trauma force to the chest wall. As a result, we frequently see associate chest wall injuries with multiple rib fractures or even flail chest wall segments. ${ }^{2,3}$ In penetrating chest wall trauma, the injury to the chest wall is more focal and often associated with only one or two rib fractures or no rib fractures at all. In most cases, the chest wall stability and physiologic function remain intact. However, some patients with thoracic gunshot wounds require a thoracotomy to control hemorrhage or repair airway or esophageal injuries. The chest wall stability is usually restored with an appropriate surgical closure of the thorax. The blunt pulmonary contusion is also associated with injuries to abdominal organs such as the spleen, liver, and intestine requiring laparotomy. This additional injury burden likely affects the need for mechanical ventilation, complications, outcome and overall mortality. This is also reflected in this NTDB data set with a higher injury severity score in the closed pulmonary contusion versus open pulmonary contusion group.

Both injury patterns are associated with diaphragmatic injuries. Diaphragmatic lacerations in blunt trauma tend to be larger defects compared to penetrating injuries. Both requiring surgical repair, however, more substantial defects may result in decreased diaphragmatic function after repair prolonging the process of weaning off mechanical ventilation.

The volume of lung parenchyma affected by contusion may also be an important factor. Deunk et al. demonstrated that pulmonary contusion visible on both CT scan and chest X-ray are associated with a higher mortality rate compared to occult pulmonary contusion visible only on CT scan but not on chest X-ray. ${ }^{4}$ Closed pulmonary contusion in blunt trauma may be associated with a larger lung volume affected by contusion compared to penetrating trauma-related open pulmonary contusions (Figs. 2 and 3). Three-dimensional volume measurements from CT scan data of pulmonary contusion patients are not reported in the literature and further research in that area is required to better understand the relationship between affected lung volume by contusion and associated clinical management and outcome.

Pulmonary contusion alternates the immune function of lymphocyte and macrophage cells and by this mechanism, increases the risk of sepsis and mortality. ${ }^{6,7}$ This might be a potential reason why pulmonary contusion with blunt trauma has an increased pneumonia rate and mortality.

A critical review of the study populations reveals three important differences between the two groups closed versus open pulmonary contusion. The injury severity score was significantly higher in the closed pulmonary contusion group with 21.6 versus 18.6 . This might be secondary to the associated chest wall and abdominal injuries, which may influence the complication rate and outcome. Furthermore, the closed pulmonary contusion group was significantly older, and the male/female ratio was higher in the open pulmonary contusion group. Traumatic brain injuries and spinal cord injuries may affect the length of mechanical ventilation and ICU stay in the blunt pulmonary contusion patient population, however available information in this data set does not allow conclusions on their interaction. These differences may further affect the complication rate and outcome.

In summary, pulmonary contusion is a common disease in patients with thoracic trauma. About onethird of these patients have associated respiratory failure requiring mechanical ventilation. Closed pulmonary contusion secondary to blunt trauma compared to open pulmonary is associated with higher injury severity score, increased ventilator days, longer ICU and hospital stay, higher pneumonia rate, and increased mortality. Further research is needed to better understand the underlying pathophysiology and to optimize the clinical management. Open pulmonary contusion secondary to blast effect from gunshot wounds might be a very different entity then closed pulmonary contusions secondary to blunt trauma. Associated operative interventions like thoracotomy may also affect length of mechanical ventilation and ICU stay, however further detailed conclusions cannot be drawn from this dataset. Pulmonary contusions are associated with a significant mortality rate and further work is needed to better understand the associated inflammatory and immune response. Careful intensive care unit management, lung protective ventilation strategies, ${ }^{8,9}$ and closed surveillance for signs of pneumonia may allow to further decrease the high mortality rate. 

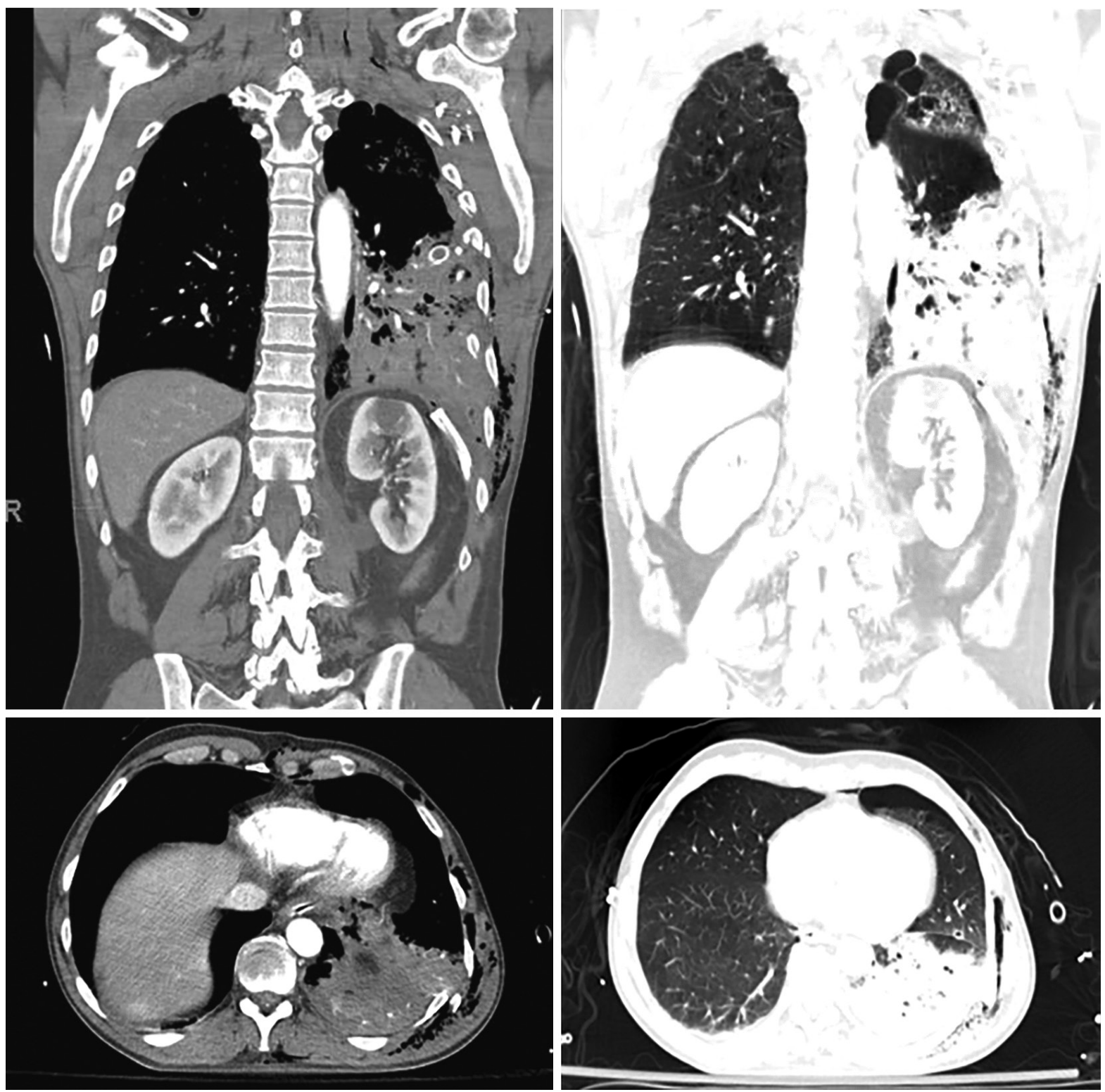

Fig. 1: Closed pulmonary contusion in a 58-year-old male status post motor vehicle accident. The patient had associated multiple rib fractures, pneumothorax, diaphragmatic laceration and spleen laceration requiring exploratory laparotomy diaphragmatic repair and splenectomy.
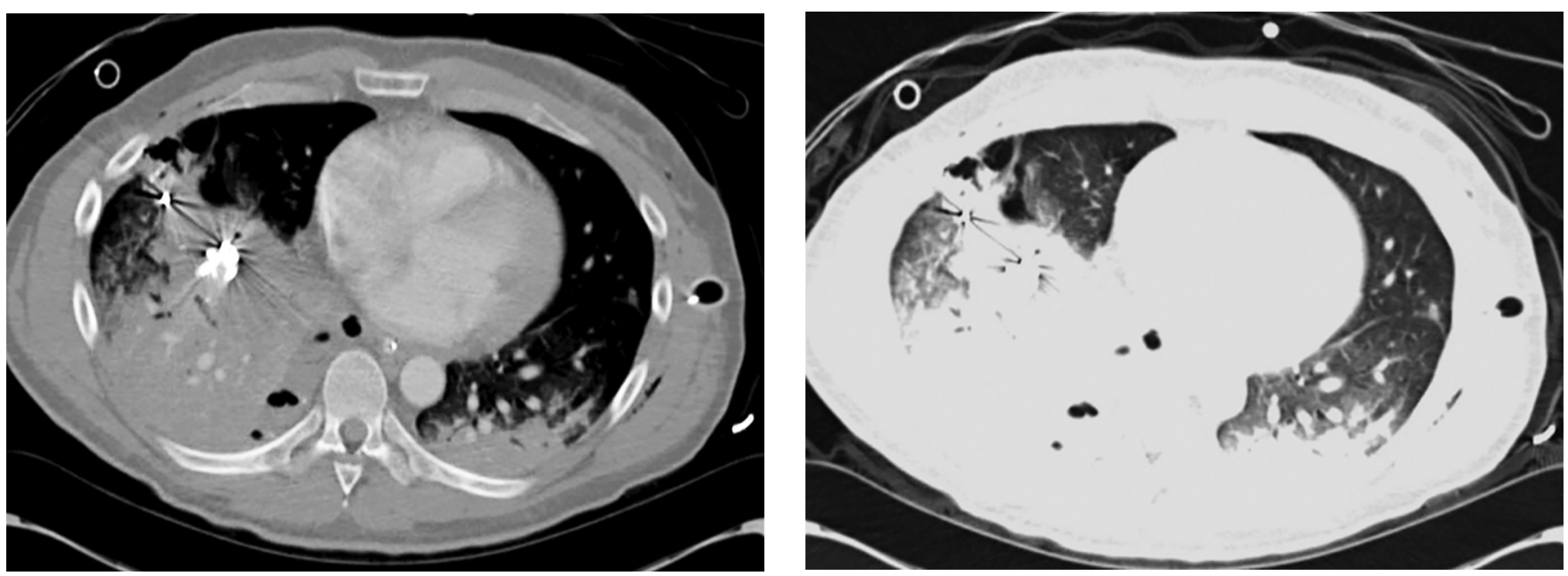

Fig. 2: A 37-year-old male status post guns shot wound the right chest with significant pulmonary contusion secondary to blast effect. The chest wall injury is minimal compared to the blunt trauma case in Figure 1. 


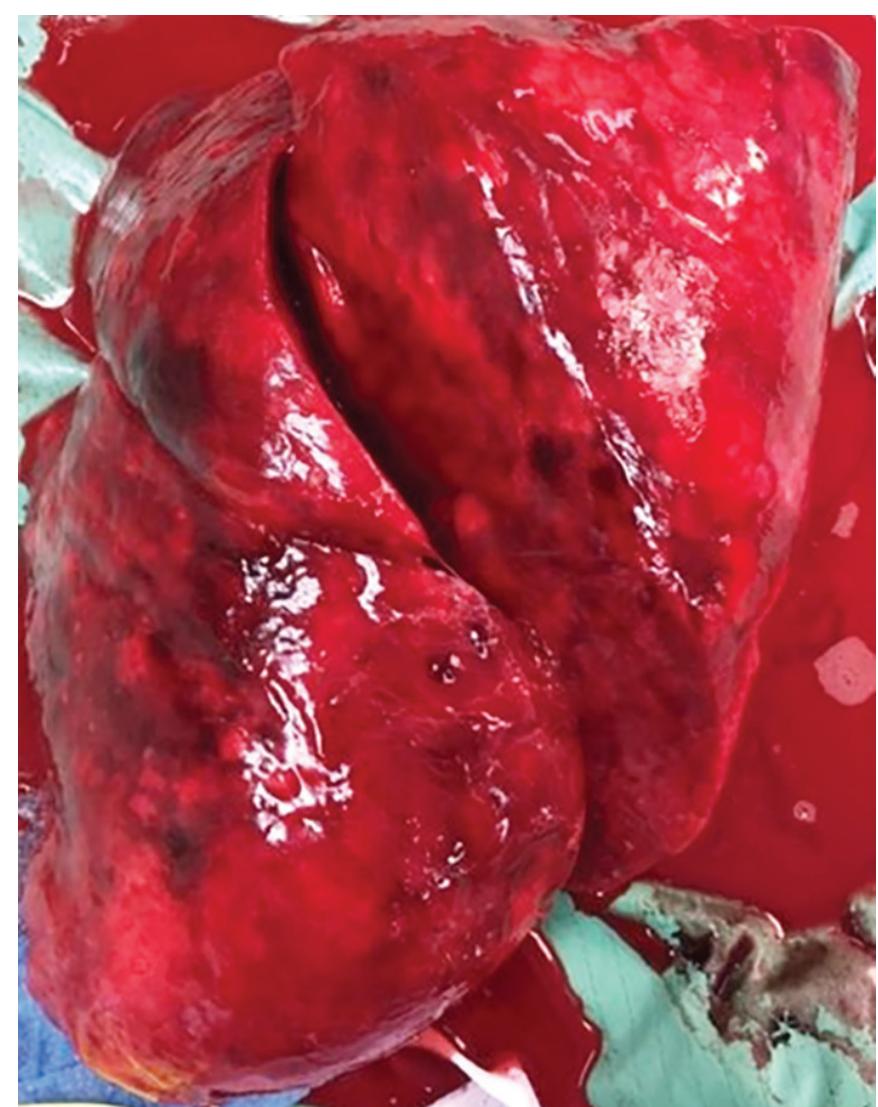

Fig. 3: Severe blunt pulmonary contusion of a right vehicle accident complete avulsion of lung hilum requiring pneumo-nectomy in a 15-year-old male patient status post motor vehicle accident.

\section{REFERENCES}

1. Cohn SM. Pulmonary contusion: Review of the clinical entity. J Trauma. 1997;42(5):973-979.

2. Simon B, Ebert J, Bokhari F, Capella J, Emhoff T, Hayward $\mathrm{T}, 3 \mathrm{rd}$, et al. Management of pulmonary contusion and flail chest: An Eastern Association for the Surgery of Trauma practice management guideline. J Trauma Acute Care Surg. 2012;73(5 Suppl 4):S351-S361.

3. Battle CE, Evans PA. Predictors of mortality in patients with flail chest: A systematic review. Emerg Med J. 2015;32(12):961-965.

4. Deunk J, Poels TC, Brink M, Dekker HM, Kool DR, Blickman JG, et al. The clinical outcome of occult pulmonary contusion on multidetector-row computed tomography in blunt trauma patients. J Trauma. 2010;68(2):387-394.

5. Surgeons ACo. National Sample Program 2012 User Manual. www.ntdborg/nsp. 2013.

6. Perl M, Gebhard F, Bruckner UB, Ayala A, Braumuller S, Buttner C, et al. Pulmonary contusion causes impairment of macrophage and lymphocyte immune functions and increases mortality associated with a subsequent septic challenge. Crit Care Med. 2005;33(6):1351-1358.

7. Hoth JJ, Wells JD, Yoza BK, McCall CE. Innate immune response to pulmonary contusion: Identification of cell type-specific inflammatory responses. Shock. 2012;37(4):385-391.

8. Cohn SM, Dubose JJ. Pulmonary contusion: An update on recent advances in clinical management. World J Surg. 2010;34(8):1959-1970.

9. Allen GS, Coates NE. Pulmonary contusion: A collective review. Am Surg. 1996;62(11):895-900. 


\section{INVITED COMMENTARY}

Chest trauma continues to be a common challenge that is confronted by virtually all professionals involved with the early resuscitation of injured patients. The present study from colleagues from Ryder Trauma Centre at Jackson Memorial in Miami reminds us that lung contusions remain a heterogeneous group, with many obvious distinctions that vary according to the mechanisms of injury. The study was designed to find the differences between lung contusions caused by blunt (closed) versus penetrating (open) injuries. It is worthwhile reading.

The authors analyzed a large cohort of almost 13.000 injured patients with the lung contusion, from the NTDB in 2012. That year the NTDB cataloged almost 180.000 patients. Blunt lung contusions were registered in 12.329 while penetrating contusions were diagnosed in only 555 patients.

The first conclusion is an easy one: high-energy blunt lung contusions are much more common and occur in more than $7 \%$ of all injured patients. Further analysis revealed that patients with blunt lung contusions are older, have the higher burden of injury (ISS), require longer ICU and hospital stay, longer mechanical ventilation, complicate more (more pneumonia) and die more (significantly higher mortality of $7.6 \%$ vs. $4.6 \%$ for penetrating). In my opinion, the second conclusion is also easy: do not underestimate lung contusions, in particular when blunt mechanisms cause them.

The Discussion presents a good conversation on the reasons for the differences between the two groups. It is possible that head injury (not investigated in this study), was responsible for many of the deaths in the group of patients with the blunt lung contusion. So were the extra-thoracic injuries, not the lung contusion itself, responsible for the difference in mortality in many patients. Another explanation is the amount of lung contused by the high-energy impact versus a single bullet, leading more frequently to pneumonia, requiring longer mechanical ventilation and also being responsible for more deaths.

This is a good manuscript that reminds us not to underestimate lung contusions, in particular in patients suffering blunt injuries.

Sandro Rizoli

Professor, Surgery and Critical Care, Medicine University of Toronto Medical Director, Trauma and Acute Care Service St Michael's Hospital 